\title{
Resource Sharing in an Integrated Wireless Cellular/WLAN System
}

\author{
Enrique Stevens-Navarro and Vincent W.S. Wong \\ Department of Electrical and Computer Engineering \\ The University of British Columbia, Vancouver, Canada \\ E-mail: $\{$ enriques, vincentw\}@ece.ubc.ca
}

\begin{abstract}
The integration of cellular networks and Wireless Local Area Networks (WLANs) aims to take advantage of the coverage-complementary characteristics of both wireless networks. For applications which require quality of service $(\mathrm{Q} o S)$ guarantee (e.g., voice, real-time video), admission control is necessary so as to limit the number of connections in a network. A connection request will be blocked if the minimum bandwidth requirement cannot be satisfied. In this paper, we propose an integrated cellular/WLAN system with resource sharing capabilities. In this system, we analyze two admission control algorithms, namely: cutoff priority and fractional guard channel. The admission algorithms consider new connection requests, requests due to either horizontal handoff or vertical handoff. If one access network does not have enough resources to support a handoff connection request, the request will be transferred to another network. We propose an analytical model and determine the new connection blocking, handoff dropping probabilities of this integrated cellular/WLAN system. Results show that the performance improves significantly when resource sharing is allowed between different wireless access networks.
\end{abstract}

\section{INTRODUCTION}

The architecture for the next generation of wireless networks aims to integrate cellular networks and Wireless Local Area Networks (WLANs). There are various standardization bodies working towards this vision. Examples include the 3GPP (3rd Generation Partnership Project), 3GPP2, and the IEEE 802.21 Media Independent Handover Working Group [1]. Cellular networks provide wide coverage while WLANs provide coverage in localized hot-spot areas (i.e., heavily populated small areas with low mobility users). Furthermore, new mobile devices are envisioned to be equipped with multiple interfaces to establish connections with different types of wireless networks. As the users move within the coverage areas, they are able to switch connections among networks. The process of switching connections is called handoff. The handoff is called horizontal if it is among cells of the same network, and is called vertical if it is among cells of different access networks (e.g., from CDMA 1xRTT to a WLAN).

In order to achieve a seamless integrated cellular/WLAN system, there are several technical challenges that have to be addressed. They include mobility management, resource allocation, admission control, security, and pricing [2]. Recently, an effective admission control scheme for voice and data services in cellular/WLAN systems is proposed in [3]. The admission strategy considers the limited fractional guard channel scheme. In [4], the WLAN-first scheme is also investigated for the same cellular/WLAN system. In [5], an analytical model of an integrated 3G/WLAN network is proposed and the new call bounding admission scheme is considered. The ongoing connections in the WLANs are considered new connections in the $3 \mathrm{G}$ cellular system when vertical handoffs are performed. In [6], a general model for load sharing in a heterogeneous UMTS/WLAN system is proposed to increase the capacity of the cellular network. It is assumed that connections cannot be switched from one network to another during the lifetime of a connection. In [7], vertical handoffs are used to balance the traffic load, and to manage radio resources efficiently among access networks.

Currently, there is a need to extend, and to develop new models to facilitate the performance evaluation of cellular networks and WLANs working together. To this end, in this paper, we propose a model of an integrated cellular/WLAN system with resource sharing and admission control capabilities. The objective is to improve the connection-level performance of the system. Handoffs are assumed to have priority over new connections. If one access network does not have enough resources to support a handoff connection request, the request will be transferred to another network. Two admission control strategies, namely: cutoff priority and fractional guard channel, are used in the cellular/WLAN system. The new connection blocking and handoff dropping probabilities are evaluated by using an iterative algorithm based on the Erlang fixed-point approximation [8].

The contribution of this paper is an analytical model of an integrated cellular/WLAN system with admission control and resource sharing capabilities that is able to effectively use the additional capacity provided by the WLANs. This paper is organized as follows. The integrated cellular/WLAN model is described and analyzed in Section II. Numerical results are presented in Section III. Conclusions are given in Section IV.

\section{Cellular/Wlan System Model}

We consider an integrated cellular/WLAN system where one or more WLANs may be deployed inside each cell of the cellular system. The cellular network and WLANs have an agreement to share resources (i.e., unused capacity) to guarantee the connection-level QoS, and to reduce the handoff dropping probability. In this system, there are two specific 


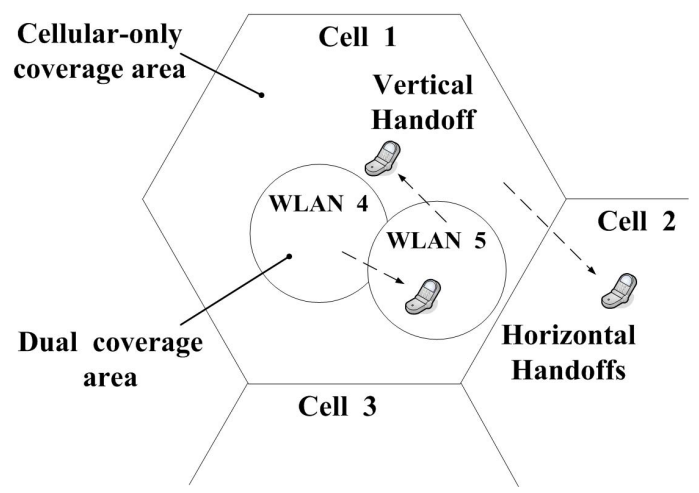

Fig. 1. Integrated Wireless Cellular/WLAN system.

coverage areas to consider, namely: the cellular-only area, and the dual-coverage area. These areas are shown in Fig. 1. In this context, coverage means service availability. Horizontal and vertical handoffs can occur in different coverage areas.

We consider a cellular system with $M^{c}$ cells. Let $\boldsymbol{A}_{i}^{c}$ be the set of cells adjacent to cell $i, \boldsymbol{W}_{i}^{c}$ be the set of WLANs inside the coverage of cell $i, \boldsymbol{A}_{\boldsymbol{k}}^{\boldsymbol{w}}$ be the set of WLANs adjacent to WLAN $k$, and $D_{k}^{w}$ be the set containing the overlaying cell of WLAN $k$ (i.e., a dual-coverage area). As an example, from Fig. 1, we have that $M^{c}=3, \boldsymbol{A}_{1}^{c}=\{2,3\}$, $\boldsymbol{W}_{\mathbf{1}}^{\boldsymbol{c}}=\{4,5\}, \boldsymbol{A}_{\mathbf{4}}^{\boldsymbol{w}}=\{5\}$, and $\boldsymbol{D}_{\mathbf{4}}^{\boldsymbol{w}}=\{1\}$. The new connection arrival processes to cell $i$ and WLAN $k$ are Poisson with rates $\lambda_{i}^{c}$ and $\lambda_{k}^{w}$, respectively, which are independent of other arrival processes. The channel holding time of a connection in cell $i$ (i.e., the time that a user is using resources in cell $i$ ) is an exponentially distributed random variable with mean $1 / \mu_{i}^{c}$. The channel holding time in WLAN $k$ is exponentially distributed with mean $1 / \mu_{k}^{w}$. Both are independent of earlier arrival times and connection duration times.

At the end of a holding time, a connection in cell $i$ of the cellular system may terminate and leave the system with probability $q_{i T}^{c}$, or move within the system and continue in an adjacent cell or WLAN with probability $1-q_{i T}^{c}$. The probability that a connection continues and moves to an adjacent cell of cell $i$ or WLAN $k$ inside cell $i$ is given by

$$
P_{i}^{c}[\text { mobility }]=1-q_{i T}^{c}=\sum_{j \in A_{i}^{c}} q_{i j}^{c}+\sum_{k \in W_{i}^{c}} q_{i k}^{c}
$$

where $q_{i j}^{c}$ is the probability to attempt a horizontal handoff to adjacent cell $j$, and $q_{i k}^{c}$ is the probability to attempt a vertical handoff to WLAN $k$ inside cell $i$.

On the other hand, at the end of a holding time of a connection in WLAN $k$, it may terminate and leave the system with probability $q_{k T}^{w}$. The probability that the connection continues and moves to the overlaying cell or to an adjacent WLAN of WLAN $k$ is given by

$$
P_{k}^{w}[\text { mobility }]=1-q_{k T}^{w}=\sum_{l \in A_{k}^{w}} q_{k l}^{w}+\sum_{i \in D_{k}^{w}} q_{k i}^{w}
$$

where $q_{k l}^{w}$ is the probability to attempt a horizontal handoff to adjacent WLAN $l$, and $q_{k i}^{w}$ is the probability of attempt a vertical handoff to overlaying cell $i$.

\section{A. Admission Control and Resource Sharing}

Each cell $i$ of the cellular system has a capacity of $C_{i}^{c}$ units of bandwidth, while each WLAN $k$ has a capacity of $C_{k}^{w}$ units of bandwidth. We define $T_{i}^{c}$ and $T_{k}^{w}$ as the reservation parameters of cell $i$ and WLAN $k$ for handoff connections, respectively. These parameters act as the admission policy to provide handoffs priority over new connections. Two admission control schemes from cellular networks [9] are considered: the cutoff priority and the fractional guard channel.

Let $n_{i}$ be the number of connections in cell $i$. When cell $i$ is in any of the states $n_{i} \leq C_{i}^{c}-T_{i}^{c}$, it accepts new and handoff connections under both admission policies. When cell $i$ is in any of the states $n_{i}>C_{i}^{c}-T_{i}^{c}$, only handoff requests are accepted for the cutoff priority scheme. For the fractional guard channel scheme, handoff requests are accepted; each new connection request is accepted with probability $\omega_{i}^{c}$. The same admission policies apply to WLANs with probability $\omega_{k}^{w}$. Note that cutoff priority is a particular case of the fractional guard channel (with $\omega_{i}^{c}=0$ ).

The resource sharing capability provided by the agreement among different access networks operates as follows: if a handoff request is not accepted in one network, then the request is transferred to the other network. In the cellular system, only when the user is inside the dual-coverage area can the connection be transferred to the WLAN. Connections that are within the WLAN can always be transferred to the corresponding overlaying cell in the cellular system.

\section{B. Traffic Equations}

The occupancy of a cell evolves according to a birthdeath process independent of other cells. The process for cell $i$ evolves with birth rate $\rho_{i}^{c}$ for the unreserved states (i.e., $0<n_{i} \leq C_{i}^{c}-T_{i}^{c}$ ), and $\alpha_{i}^{c}$ for the reserved states (i.e., $C_{i}^{c}-T_{i}^{c}<n_{i} \leq C_{i}^{c}$ ). The death rate of cell $i$ in state $n_{i}$ is $n_{i} \mu_{i}^{c}$. The total traffic offered to cell $i$ in state $n_{i}$ is

$$
\begin{gathered}
\rho_{i}^{c}=\lambda_{i}^{c}+\sum_{j \in A_{i}^{c}} v_{j i}^{c}+\sum_{k \in W_{i}^{c}} v_{k i}^{w}+\sum_{l \in W_{i}^{c}} \gamma_{l i}^{w}, n_{i} \leq C_{i}^{c}-T_{i}^{c}, \quad \text { (3) } \\
\alpha_{i}^{c}=\lambda_{i}^{c} \omega_{i}^{c}+\sum_{j \in A_{i}^{c}} v_{j i}^{c}+\sum_{k \in W_{i}^{c}} v_{k i}^{w}+\sum_{l \in W_{i}^{c}} \gamma_{l i}^{w}, \quad n_{i}>C_{i}^{c}-T_{i}^{c},
\end{gathered}
$$

with $\omega_{i}^{c}=0$ for the cutoff priority scheme, and $0 \leq \omega_{i}^{c} \leq 1$ for the fractional guard channel scheme. In (3) and (4), the term $v_{j i}^{c}$ is the horizontal handoff rate of cell $j$ offered to cell $i$, for adjacent cells $i$ and $j$, and is given by

$$
\begin{aligned}
v_{j i}^{c}= & \lambda_{j}^{c}\left(1-B_{j}^{c}\right) q_{j i}^{c}+\sum_{x \in A_{j}^{c}} v_{x j}^{c}\left(1-B_{h_{j}}^{c}\right) q_{j i}^{c}+ \\
& \sum_{y \in W_{j}^{c}} v_{y j}^{w}\left(1-B_{h_{j}}^{c}\right) q_{j i}^{c}+\sum_{z \in W_{j}^{c}}\left[\gamma_{z j}^{w}\left(1-B_{h_{j}}^{c}\right) q_{j i}^{c}\right],
\end{aligned}
$$

where $B_{j}^{c}$ and $B_{h_{j}}^{c}$ are the new connection blocking and handoff dropping probabilities in cell $j$, respectively. 
In (3)-(5), $\gamma_{z j}^{w}$, corresponds to all handoff traffic that is not accepted in WLAN $z$ and hence transferred to cell $j$, then

$$
\gamma_{z j}^{w}=v_{j z}^{w} B_{h_{z}}^{w}+\sum_{l \in A_{z}^{w}} v_{l z}^{w} B_{h_{z}}^{w} .
$$

The term $v_{k i}^{w}$ is the vertical handoff rate of WLAN $k$ offered to overlay cell $i$, and is given by

$$
\begin{aligned}
v_{k i}^{w}= & \lambda_{k}^{w}\left(1-B_{k}^{w}\right) q_{k i}^{w}+\sum_{x \in A_{k}^{w}} v_{x k}^{w}\left(1-B_{h_{k}}^{w}\right) q_{k i}^{w}+ \\
& \sum_{y \in D_{k}^{w}} v_{y k}^{w}\left(1-B_{h_{k}}^{w}\right) q_{k i}^{w}+\sum_{z \in D_{k}^{w}}\left[\zeta_{z k}^{c}\left(1-B_{h_{k}}^{w}\right) q_{k i}^{w}\right],
\end{aligned}
$$

where $B_{k}^{w}$ and $B_{h_{k}}^{w}$ are the new connection blocking and handoff dropping probabilities in WLAN $k$, respectively.

In (7), $\zeta_{z k}^{c}$ is the proportion of all handoff traffic that is not accepted in cell $z$ and hence transferred to WLAN $k$, then

$$
\zeta_{z k}^{c}=\left(v_{k z}^{w} B_{h_{z}}^{c}+\sum_{l \in A_{z}^{c}} v_{l z}^{c} B_{h_{z}}^{c}\right) R_{z k}
$$

where $R_{z k}$ is the coverage factor between WLAN $k$ and overlay cell $z$. The coverage factor $R_{z k}$ considers the coverage ratio between the radio coverage area of WLAN $k$ and the radio coverage area of cell $z$ with $0<R_{z k} \leq 1$.

From the process cell $i$, the detailed balance equations are

$$
\begin{aligned}
& P_{i}^{c}\left(n_{i}-1\right) \rho_{i}^{c}=P_{i}^{c}\left(n_{i}\right) n_{i} \mu_{i}^{c} \quad 0 \leq n_{i} \leq C_{i}^{c}-T_{i}^{c}, \\
& P_{i}^{c}\left(n_{i}-1\right) \alpha_{i}^{c}=P_{i}^{c}\left(n_{i}\right) n_{i} \mu_{i}^{c} \quad C_{i}^{c}-T_{i}^{c}<n_{i} \leq C_{i}^{c},
\end{aligned}
$$

where $P_{i}^{c}\left(n_{i}\right)$ is the stationary distribution that cell $i$ is in state $n_{i}$, and is given by

$$
\begin{gathered}
P_{i}^{c}\left(n_{i}\right)=\frac{\left(\rho_{i}^{c}\right)^{n_{i}}}{G_{i}^{c} n_{i} !\left(\mu_{i}^{c}\right)^{n_{i}}}, \quad n_{i} \leq C_{i}^{c}-T_{i}^{c}, \\
P_{i}^{c}\left(n_{i}\right)=\frac{\left(\rho_{i}^{c}\right)^{C_{i}^{c}-T_{i}^{c}}\left(\alpha_{i}^{c}\right)^{n_{i}-C_{i}^{c}+T_{i}^{c}}}{G_{i}^{c} n_{i} !\left(\mu_{i}^{c}\right)^{n_{i}}}, \quad n_{i}>C_{i}^{c}-T_{i}^{c}, \\
G_{i}^{c}=\sum_{n_{i}=0}^{C_{i}^{c}-T_{i}^{c}} \frac{1}{n_{i} !}\left(\frac{\rho_{i}^{c}}{\mu_{i}^{c}}\right)^{n_{i}}+ \\
\sum_{n_{i}=C_{i}^{c}-T_{i}^{c}+1}^{C_{i}^{c}} \frac{\left(\rho_{i}^{c}\right)^{C_{i}^{c}-T_{i}^{c}}\left(\alpha_{i}^{c}\right)^{n_{i}-C_{i}^{c}+T_{i}^{c}}}{n_{i} !\left(\mu_{i}^{c}\right)^{n_{i}}} .
\end{gathered}
$$

Similarly, the occupancy of WLAN $k$ evolves with birth rates $\rho_{k}^{w}$ and $\alpha_{k}^{w}$ based on the state $m_{k}$, and death rate $m_{k} \mu_{k}^{w}$. The total traffic offered to WLAN $k$ in state $m_{k}$ is

$\rho_{k}^{w}=\lambda_{k}^{w}+\sum_{l \in A_{k}^{w}} v_{l k}^{w}+\sum_{j \in D_{k}^{w}} v_{j k}^{w}+\sum_{g \in D_{k}^{w}} \zeta_{g k}^{c}, \quad m_{k} \leq C_{k}^{w}-T_{k}^{w}$,

$\alpha_{k}^{w}=\lambda_{k}^{w} \omega_{k}^{w}+\sum_{l \in A_{k}^{w}} v_{l k}^{w}+\sum_{j \in D_{k}^{w}} v_{j k}^{w}+\sum_{g \in D_{k}^{w}} \zeta_{g k}^{c}, \quad m_{k}>C_{k}^{w}-T_{k}^{w}$
In (11) and (12), the term $v_{l k}^{w}$ is the horizontal handoff rate of WLAN $l$ offered to WLAN $k$, for adjacent WLANs $l$ and $k$, and is given by

$$
\begin{aligned}
v_{l k}^{w}= & \lambda_{l}^{w}\left(1-B_{l}^{w}\right) q_{l k}^{w}+\sum_{x \in A_{l}^{w}} v_{x l}^{w}\left(1-B_{h_{l}}^{w}\right) q_{l k}^{w}+ \\
& \sum_{y \in D_{l}^{w}} v_{y l}^{w}\left(1-B_{h_{l}}^{w}\right) q_{l k}^{w}+\sum_{z \in D_{l}^{w}}\left[\zeta_{z l}^{c}\left(1-B_{h_{l}}^{w}\right) q_{l k}^{w}\right] .
\end{aligned}
$$

The term $v_{j k}^{w}$ is the vertical handoff rate of cell $j$ offered to WLAN $k$, for overlaying cells $j$ and $k$, and is given by

$$
\begin{aligned}
v_{j k}^{w}= & \lambda_{j}^{c}\left(1-B_{j}^{c}\right) R_{j k} q_{j k}^{c}+\sum_{x \in A_{j}^{c}} v_{x j}^{c}\left(1-B_{h_{j}}^{c}\right) R_{j k} q_{j k}^{c}+ \\
& \sum_{y \in W_{j}^{c}} v_{y j}^{w}\left(1-B_{h_{j}}^{c}\right) R_{j k} q_{j k}^{c}+\sum_{z \in W_{j}^{c}}\left[\gamma_{j z}^{w}\left(1-B_{h_{j}}^{c}\right) q_{j k}^{c}\right] .
\end{aligned}
$$

Due to the lack of space, we point out that the stationary distribution that WLAN $k$ is in state $m_{k}, P_{k}^{w}\left(m_{k}\right)$ is similar to equations (9)-(10), but with WLAN parameters $\rho_{k}^{w}, \alpha_{k}^{w}, \mu_{k}^{w}$, $C_{k}^{w}, T_{k}^{w}$ and $G_{k}^{w}$.

\section{Performance Measures}

In the cellular system, using the stationary distribution, the new connection blocking probability $B_{i}^{c}$ in cell $i$ is

$$
B_{i}^{c}=\sum_{n_{i}=C_{i}^{c}-T_{i}^{c}}^{C_{i}^{c}-1} P_{i}^{c}\left(n_{i}\right)\left(1-\omega_{i}^{c}\right)+P_{i}^{c}\left(C_{i}^{c}\right),
$$

and the handoff dropping probability $B_{h_{i}}^{c}=P_{i}^{c}\left(C_{i}^{c}\right)$.

Similarly, $B_{k}^{w}$ in WLAN $k$ is

$$
B_{k}^{w}=\sum_{m_{k}=C_{k}^{w}-T_{k}^{w}}^{C_{k}^{w}-1} P_{k}^{w}\left(m_{k}\right)\left(1-\omega_{k}^{w}\right)+P_{k}^{w}\left(C_{k}^{w}\right),
$$

and $B_{h_{k}}^{w}=P_{k}^{w}\left(C_{k}^{w}\right)$.

\section{Erlang Fixed-Point Approximation}

To compute the blocking/dropping probabilities in the cellular/WLAN system, the following iterative algorithm based on the Erlang fixed-point approximation [8] is used:

1) Specify $\varepsilon>0$, and set $B_{i}^{c}=B_{h_{i}}^{c}=B_{k}^{w}=B_{h_{k}}^{w}=0$.

2) Solve the system of equations of handoff rates given by (5), (7), (13), and (14).

3) Compute total traffic offered to cell $i$ from (3)-(4).

4) Compute total traffic offered to WLAN $k$ from (11)-(12).

5) Calculate blocking/dropping probabilities $B_{i(\text { new })}^{c}$, $B_{h_{i}(\text { new })}^{c}, B_{k(\text { new })}^{w}$, and $B_{h_{k}(\text { new })}^{w}$ as in Section II.C.

6) If $\left\|B_{i}^{c}\right\|+\left\|B_{h_{i}}^{c}\right\|<\varepsilon$ and $\left\|B_{k}^{w}\right\|+\left\|B_{h_{k}}^{w}\right\|<\varepsilon$, go to step 7. Otherwise, set $B_{i}^{c}=B_{i(\text { new })}^{c}, B_{h_{i}}^{c}=B_{h_{i}(\text { new })}^{c}$, $B_{k}^{w}=B_{k(\text { new })}^{w}, B_{h_{k}}^{w}=B_{h_{k}(\text { new })}^{w}$, and return to step 2 .

7) Stop.

In this paper, the function $\|\cdot\|$ is defined as $\sum_{j}\left|B_{j(\text { new })}-B_{j}\right|$. We use $\varepsilon=10^{-8}$, and the convergence of the algorithm is obtained after a few iterations. 


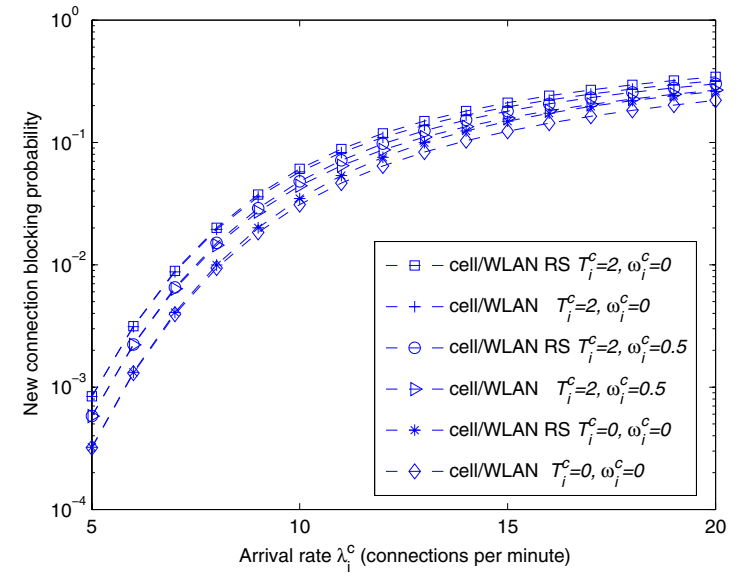

Fig. 2. New connection blocking probability.

\section{NumERICAL RESULTS}

We evaluate a wireless system consisting of a cellular network with $M^{c}=3$, and $\left|W_{i}^{c}\right|=2$ as shown in Fig. 1. We compare the cellular/WLAN system with and without resource sharing. We assume that all cells in the cellular network have capacity $C_{i}^{c}=30$ units of bandwidth, and all WLANs have capacity $C_{k}^{w}=60$. Channel holding times in the cells of the cellular network have means $1 / \mu_{i}^{c}=1 \mathrm{~min}$, and channel holding times in the WLANs have means $1 / \mu_{k}^{w}=4 \mathrm{~min}$. The coverage factor $R_{j k}$ is set to 0.75 . The mobility of users given by (1) in the cellular network is $q_{i T}^{c}=0.4$, and the mobility in the WLANs, given by (2) is $q_{k T}^{w}=0.4$. These values define a mobility level of $60 \%$ (i.e., $60 \%$ of users perform handoffs).

Fig. 2 shows the new connection blocking probability at cell-level in the integrated cellular/WLAN system when the arrival rate $\lambda_{i}^{c}$ is increased from 5 to 20 connections per minute in all cells, and $\lambda_{k}^{w}=5$. The results labeled as $T_{i}^{c}=2$, $\omega_{i}^{c}=0$ correspond to the admission policy cutoff priority, $T_{i}^{c}=2, \omega_{i}^{c}=0.5$ to the fractional guard channel, and $T_{i}^{c}=0, \omega_{i}^{c}=0$ when no admission policy is used. Note that the cellular/WLAN system achieves very close blocking probabilities with and without resource sharing. Recall that the resource sharing is only for handoffs. As expected, the use of admission control increases the probability of blocking new connections at the expense of a lower handoff dropping probability. The highest blocking probabilities are achieved by cutoff priority because it rejects all new connections when the cell is in the reserved states for handoffs, while fractional guard channel accept them with probability $\omega_{i}^{c}=0.5$.

Fig. 3 shows the handoff dropping probability at cell-level in the integrated cellular/WLAN system for the same increase in the arrival rates. This is the handoff dropping probability for any handoff (i.e., horizontal or vertical) within the dualcoverage area. The integrated cellular/WLAN with resource sharing achieves lower handoff dropping probabilities due to the additional capacity shared by the WLANs in the dualcoverage areas. In this case, the highest dropping probabilities in both systems are achieved when no admission control is used, and the lowest when cutoff priority is used. By using

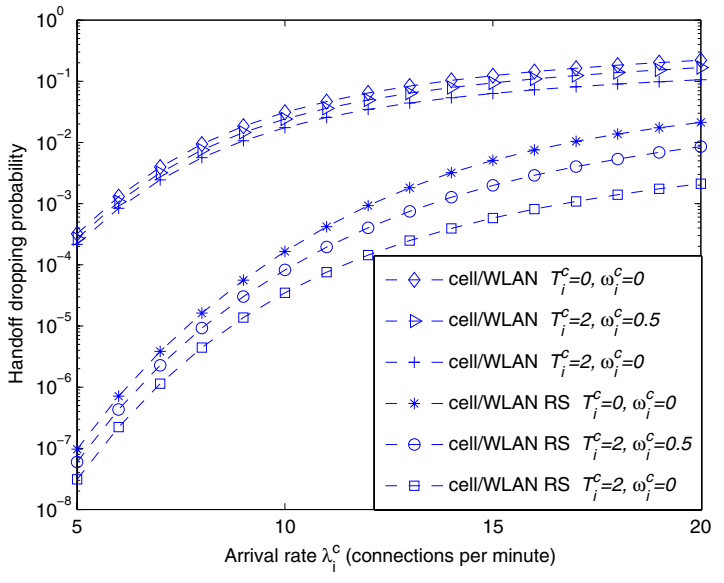

Fig. 3. Handoff dropping probability.

reservation parameters $T_{i}^{c}=T_{k}^{w}=2$, the handoff dropping probability is reduced by $58 \%$ at an expense of a $30 \%$ increase in the new connection blocking probability. Note that the admission policy, and the reservation parameters $\left(T_{i}^{c}, T_{k}^{w}\right)$ have to be selected carefully in order to meet the desired connection-level QoS.

\section{Conclusions}

In this paper, we presented an analytical model of an integrated cellular/WLAN system with admission control and resource sharing capabilities that is able to effectively use the additional capacity provided by the WLANs. Results show that the performance improves significantly when resource sharing is allowed between different wireless access networks.

\section{ACKNOWLEDGMENT}

This work was supported by Bell Canada, Natural Sciences and Engineering Research Council of Canada, and the Programa de Mejoramiento del Profesorado (PROMEP) from Mexico.

\section{REFERENCES}

[1] IEEE 802.21 Working Group, http://www.ieee802.org/21/.

[2] W. Song, H. Jiang, W. Zhuang, and X. Shen, "Resource Management for QoS Support in Cellular/WLAN Interworking," IEEE Network, vol. 19, no. 5, pp. 12-18, September/October 2005.

[3] W. Song and W. Zhuang, "QoS Provisioning via Admission Control in Cellular/Wireless LAN Interworking," in Proc. of BROADNETS'05, Boston, MA, October 2005

[4] W. Song, H. Jiang, W. Zhuang, and A. Saleh, "Call Admission Control for Integrated Voice/Data Services Cellular/WLAN Interworking," in Proc. of IEEE ICC'06, Istanbul, Turkey, June 2006.

[5] S. Tang and W. Li, "Performance Analysis of the 3G Network with Complementary WLANs," in Proc. of IEEE GLOBECOM'05, St. Louis, MO, November 2005.

[6] D. Chen, X. Wang, and A. Elhakeem, "Load Sharing with Buffering over Heterogeneous Networks," in Proc. of IEEE VTC'05 Fall, Dallas, TX, September 2005.

[7] X. Liu, V. Li, and P. Zhang, "Joint Radio Resource Management through Vertical Handoffs in 4G Networks," in Proc. of IEEE GLOBECOM'06, San Francisco, CA, November 2006.

[8] K. Ross, Multiservice Loss Models for Broadband Telecommunication Networks. Springer, 1995.

[9] Y. Fang and Y. Zhang, "Call Admission Control Schemes and Performance Analysis in Wireless Mobile Networks," IEEE Transactions on Vehicular Technology, vol. 51, no. 2, pp. 371-382, March 2002. 